Revue d'histoire de l'Amérique française

REVUE D.HISTOIRE DE L'AMÉRIQUE FRANÇAISE

\title{
FILION, Mario, Jean-Charles FORTIN, Roland VIAU et Pierre LAMBERT, Histoire du Haut-Saint-Laurent (Sainte-Foy, Institut québécois de recherche surr la culture, 2000), 439 p.
}

\section{André LaRose}

Volume 55, numéro 2, automne 2001

URI : https://id.erudit.org/iderudit/010371ar

DOI : https://doi.org/10.7202/010371ar

Aller au sommaire du numéro

Éditeur(s)

Institut d'histoire de l'Amérique française

ISSN

0035-2357 (imprimé)

1492-1383 (numérique)

Découvrir la revue

Citer ce compte rendu

LaRose, A. (2001). Compte rendu de [FILION, Mario, Jean-Charles FORTIN, Roland VIAU et Pierre LAMBERT, Histoire du Haut-Saint-Laurent (Sainte-Foy, Institut québécois de recherche sur la culture, 2000), 439 p.] Revue d'histoire de l'Amérique française, 55(2), 265-268. https://doi.org/10.7202/010371ar d'utilisation que vous pouvez consulter en ligne.

https://apropos.erudit.org/fr/usagers/politique-dutilisation/ 
an alternative civic nationalism» (p. 316), la population «canadienne» devenant ainsi «an administrative abstraction laid on top of identities defined otherwise». On découvre alors que le seul recensement scientifique de cette période aurait ainsi raté un des objectifs centraux de l'exercice, soit définir une population sur la base nationale... Le problème vient du fait que la définition des origines est au cœur du processus identitaire à l'époque, et que ce phénomène n'a que très peu à voir avec les ambitions ultramontaines ( $c f$. les travaux de R. Larue sur ce point). Que cela plaise ou non aux contempteurs des "nationalistes » québécois, ce pays est né de cette diversité avouée et perçue des origines. Attendre que, quatre ans après la création du pays, le recensement adopte une telle catégorie identitaire dans la définition des «origines» est pour le moins surprenant...

Malgré ce curieux faux pas analytique, Politics of Population est un livre remarquable, qui permet de pousser beaucoup plus loin la réflexion sur le politique au cours des grandes transitions du $\mathrm{xIX}^{\mathrm{e}}$ siècle.

JEAN-MARIE FECTEAU

Département d'histoire Université du Québec à Montréal

FILION, Mario, Jean-Charles FORTIN, Roland VIAU et Pierre LAMBERT, Histoire du HautSaint-Laurent (Sainte-Foy, Institut québécois de recherche sur la culture, 2000), 439 p.

L'Histoire du Haut-Saint-Laurent est un ouvrage de synthèse qui présente une vue d'ensemble du milieu naturel, de la préhistoire et de l'histoire des municipalités régionales de comté (MRC) Vaudreuil-Soulanges, sur la rive nord du fleuve, Beauharnois-Salaberry et le Haut-Saint-Laurent, sur la rive sud. Douzième titre de la collection "Les régions du Québec", qui, à terme, devrait en compter plus de 20 , ce livre est le deuxième élément d'une trilogie consacrée à la Montérégie. Il se divise en 11 chapitres thématiques qui cachent un découpage chronologique invisible à prime abord, car il n'y a pas de dates dans les titres de chapitres : de la préhistoire à 1850 (chapitres 2 à 5) et de 1850 à nos jours (chapitres 6 à 11).

Après une présentation des divers éléments du milieu naturel, Pierre Lambert décrit, dans le chapitre 1, les «signes d'une région usée, marquée par une exploitation abusive du milieu » (p. 37). Roland Viau présente ensuite des «images de la préhistoire du Haut-Saint-Laurent» — un terri- 
toire occupé depuis 8000 à 8500 ans — avant de décrire la vie en Laurentie iroquoienne à la fin de la préhistoire. Mario Filion enchaîne avec trois chapitres sur la période 1600-1850 : «La conquête du territoire» (évolution de la zone habitée, marche du peuplement, poussée villageoise, faits d'armes), «Les assises d'une socio-économie naissante» (les fourrures, l'agriculture, le développement des transports, les débuts de l'industrie rurale), puis «Encadrement institutionnel et naissance de la société régionale» (les seigneuries, les Églises, le système scolaire, l'encadrement civil). Jean-Charles Fortin prend le relais et dégage les grands traits de l'évolution démographique des cent cinquante dernières années dans "Mobilité et enracinement», non sans insister sur l'importance de l'émigration. Puis il rend compte de la formidable transformation du monde du travail dans "Au champ, à l'usine et au bureau», dans un contexte où "la proximité immédiate de Montréal et le poids du voisin américain influencent le développement de l'économie haut-laurentienne sur tous les plans» (p. 209). Roland Viau poursuit avec un chapitre relatif aux «mégaprojets qui altèrent singulièrement l'écoumène de la région" au cours de "cent cinquante ans de surexploitation» (p. 241) : la construction de canaux navigables, la mise en valeur du potentiel hydraulique au moyen de barrages et de centrales hydro-électriques et l'industrialisation. Mario Filion procède ensuite à une description sommaire du réseau institutionnel avant d'esquisser le passage de la tradition à la modernité dans la société régionale. Pour terminer, Roland Viau s'attache «à des cas singuliers de l'expérience culturelle», mettant tour à tour en relief des personnalités et des entreprises collectives dans le domaine des lettres, des arts et du patrimoine.

«La constante recherche de régions administratives opérationnelles fait bien peu de cas des régions historiques » écrit J.-C. Fortin en introduction (p. 17). On en a un exemple patent dans cet ouvrage, où les anciens comtés de Châteauguay et de Huntingdon - et partant, le diocèse de Valleyfield, "premier lien organique de la société régionale» (p. 16) — sont amputés d'une partie de leur territoire (Léry, Châteauguay, Mercier et Sainte-Clotilde, d'une part, et Hemmingford, d'autre part) pour répondre, dit-on, aux vœux des Haut-Laurentiens. Kahnawake est également exclue. Résultat : la vallée de la rivière Châteauguay n’est pas envisagée comme un tout ni présentée comme une voie de pénétration dans les deux sens; les pages consacrées à «la reconquête de l'identité mohawk» ne portent que sur Akwesasne, comme si le phénomène ne touchait pas également Kahnawake, d'où sont issus les fondateurs de Saint-Régis; il n'est pas ques- 
tion du blocus du pont Mercier en 1990 ; enfin, les auteurs ont été obligés de reconstituer les statistiques historiques en fonction des limites des MRC, ce qui a forcément des conséquences sur les descriptions qui s'appuient sur ces chiffres (concernant l'étalement urbain au sud-ouest de Montréal, par exemple).

Les archives et les journaux n'ont pas été mis à profit, mais les auteurs s'appuient sur des études existantes, parfois difficiles d'accès, et exploitent abondamment les recensements canadiens publiés. Toutefois, leur histoire est essentiellement descriptive et narrative et n'ouvre guère de pistes de recherche. À l'instar des autres livres de la collection, l'ouvrage ne comporte pas de bibliographie. On se serait néanmoins attendu à y trouver un renvoi à certaines études comme l'article pénétrant d'Helen Taft Manning, «E. G. Wakefield and the Beauharnois Canal» (CHR, mars 1967), le livre de T. D. Regher à propos du scandale politico-financier entourant la construction du canal de Beauharnois dans les années 1920, The Beauharnois Scandal (1990) — question totalement passée sous silence —, et l'étude de Robert Hill au sujet de Robert Sellar, Voice of the Vanishing Minority (1998). Et à l'ère d'Internet, pourquoi pas une liste d'adresses de sites pertinents qui permettraient de se familiariser avec la région?

Amplement et judicieusement illustré, l'Histoire du Haut-Saint-Laurent comporte aussi 17 cartes et 51 tableaux. Sur ce point, plus de rigueur s'imposait. Pour produire les cartes 7 et 8 , on a utilisé trois cartes tirées de ma thèse de doctorat, mais, ce faisant, on a mal tracé certaines limites internes de la seigneurie de Beauharnois et surtout, à la carte 8 , le cartographe a commis de nombreuses erreurs de transcription dans la désignation des terres soumises à la commutation de tenure. Les travaux de Serge Courville ne sont pas cités fidèlement non plus : à la carte 6, ce n'est pas SainteClotilde au complet qui devrait être attribuée au canton de Sherrington mais seulement quelques terres à l'angle sud-est de la seigneurie de Beauharnois; enfin, à la carte 9, on a oublié Howick et inversé les cercles noirs et les cercles blancs sur la rive sud, ce qui fausse complètement la chronologie de l'origine des villages.

Premier travail de synthèse consacré au sud-ouest du Québec, l'Histoire du Haut-Saint-Laurent est, malgré les erreurs de détail, un livre équilibré, qui a le mérite de mettre en place les principaux éléments de l'histoire de la région - une région de frontière ou de passage, soumise à toutes les influences et intimement liée à toutes les grandes périodes de l'histoire économique canadienne. S'il ne parvient pas à donner un sentiment d'identité régionale aux Haut-Laurentiens (ou aux habitants du Suroît?), 
l'ouvrage devrait au moins inviter à jeter un regard neuf sur un coin de pays méconnu.

ANDRÉ LaROSE Hull, Québec

GIDNEY, Robert Douglas, From Hope to Harris. The Reshaping of Ontario's Schools (Toronto, University of Toronto, 1999), viii-362 p.

Voilà un ouvrage au titre paradoxal. Au lieu de la diatribe attendue contre les effets funestes des politiques de l'actuel Premier ministre conservateur Harris sur les écoles ontariennes, le lecteur découvre une description minutieuse et fouillée de l'influence des hauts fonctionnaires et des élus sur l'évolution du système scolaire ontarien (programme, structures administratives, financement et relations de travail) entre 1945 et 1999. Ici en effet, «Hope» ne désigne pas l'espoir (en anglais) des réformateurs progressistes brisé par la prétendue "révolution du bon sens» menée par Harris depuis 1995, mais le président de 1945 à 1950 d'une commission royale d'enquête sur les écoles ontariennes.

L'auteur, loin d'opposer Hope et Harris, les rapproche. Certes, il convient de l'incompatibilité du "néo-libéralisme» de ce dernier et de l'interventionnisme étatique prôné par J. A. Hope (et, noterions-nous, par Parent une décennie plus tard au Québec) en vue de moderniser l'école. Mais il s'attache davantage à la récurrence des discours et des actes suscités par les problèmes découlant des réformes - qu'il juge à la fois nécessaires, inachevées et chères - entreprises pour tenir compte des bouleversements démographiques, communicationnels, occupationnels, etc. dont l'Ontario (comme tout l'Occident, complèterions-nous) fut le théâtre de 1945 à 1960. Par l'ampleur des investissements publics qu'ils impliquaient, ces choix accroîtraient à terme le fardeau fiscal et l'endettement de l'État provincial et soulèveraient le problème de la répartition de leurs coûts entre les divers niveaux de gouvernement... et entre les classes sociales, aurait-il pu aussi écrire.

Sous le gouvernement conservateur de Robarts (1962-1971), les ajustements des structures et des budgets éducatifs visaient surtout à bâtir de nouvelles écoles modernes et capables d'accueillir l'immense cohorte du baby-boom, à adapter l'enseignement aux besoins de la "nouvelle économie » des années 1960 et, sous la pression populaire, à démocratiser 